\title{
Coping with COVID-19: An Examination of the Role of (Non) Religiousness/(Non)Spirituality
}

\author{
Dena M. Abbott ${ }^{1}$ iD $\cdot$ Andrew S. Franks ${ }^{2,3}$ D
}

Accepted: 8 May 2021 / Published online: 22 May 2021

(c) The Author(s), under exclusive licence to Springer Science+Business Media, LLC, part of Springer Nature 2021

\begin{abstract}
Psychological distress and coping strategies employed during collective trauma events may vary for theists and atheists, as well as others along the (non)religious spectrum. The present study explored these differences via data collected from a US-based sample during the COVID-19 pandemic. Statistical models suggested relationships between maladaptive coping and distress for all participants and potential differences in coping and, in turn, distress between participants high and low in institutional religiousness and individual spirituality. Additionally, all participants, though especially nonreligious participants, appeared less able to engage in adaptive emotion-focused coping strategies. Implications for future research are provided.
\end{abstract}

Keywords Nonreligious $\cdot$ Coping $\cdot$ Collective trauma $\cdot$ COVID-19 pandemic

\section{Introduction}

The COVID-19 pandemic represents a global collective trauma event (Duane et al. 2020) and is among the first such events to disrupt and compromise the physical and emotional safety and lives of all United States (U.S.) Americans in the contemporary era. Due to the novel nature of the current pandemic and diverse challenges (e.g., social isolation, economic hardship, mental and physical health, grief), the utility of commonly employed coping strategies is unknown. In general, adaptive coping strategies are associated with positive outcomes, whereas maladaptive

Dena M. Abbott

dabbott5@unl.edu

Andrew S. Franks

andrew.franks@cmich.edu

1 Department of Education Psychology, University of Nebraska - Lincoln, 222 Teachers College Hall, Lincoln, NE 68588-0345, USA

2 Department of Psychology, Princeton University, Princeton, NJ, USA

3 Department of Psychology, Central Michigan University, Mt. Pleasant, MI 48859, USA 
coping strategies are associated with distress (Lazarus et al. 1984). However, extant literature exploring coping strategies used in response to large-scale disasters often emphasizes the utility of religious and spiritual coping, in particular (Aten et al. 2019), and excludes nonreligious and/or nonspiritual people (Hwang et al. 2011). Such studies may erroneously infer that nonreligious/nonspiritual people are detrimentally impacted by the absence of religious/spiritual (R/S) coping despite their omission from participation. In fact, little is known about the manner by which nonreligious and nonspiritual people cope with large-scale events with the potential for individual and collective trauma, an omission the current study sought to address.

\section{Collective Trauma \& the COVID-19 Pandemic}

Collective traumas are events that disrupt daily life for an entire community and include natural and human-caused disasters (Erickson 1976; Silver et al. 2013), as well as sociopolitical oppressions (Comas-Díaz et al. 2019; Kelly et al. 2020). These shared traumatic events often deteriorate the sense of safety and security of those affected (Silver et al. 2013) and have negative psychological consequences (Goldmann et al. 2014; Luszczynska et al. 2009). Though destruction and disruption often remain following such disasters, pandemics, including the COVID-19 pandemic, differ from other natural (e.g., weather-related) and human-made (e.g., mass shootings) disasters in the sustained nature of the event itself.

Symptoms of posttraumatic stress disorder (PTSD), including depressive and anxious symptoms, are possible in response to collective trauma (Goldmann et al. 2014) but vary in prevalence (Bonanno 2004; Neria et al. 2008). Many factors other than the traumatic event likely influence the presentation of psychological symptoms, including disruption distress, social support and coping (Baral et al. 2019). Survivors of the SARS pandemic in Hong Kong and Taiwan reported trauma-related and other psychological symptoms including hopelessness, alienation and long-term changes to mental and physical health. Similar to COVID-19, in addition to illness and death, social and economic disruption accompanied SARS (Lei et al. 2020. Though previous exposure to such collective trauma events likely increases resilience to future traumatic events (Bonanno et al. 2010), the U.S. has not been significantly disrupted by a similar pandemic (e.g., requiring physical distancing, face coverings, hand hygiene, widespread disruptions to schooling and employment) in over 100 years. Therefore, US residents may experience more psychological consequences and possess fewer coping resources than people living in countries significantly impacted by recent, similar pandemics.

\section{Coping with Traumatic Events}

Coping refers to use of processes aimed at reducing, tolerating, or mastering stress. In general (Lazarus et al. 1984), in response to natural and human-made disasters (Cherry et al. 2017; Shing et al. 2016) and in the face of other global health crises like HIV/AIDS (Blashill et al. 2011), emotion-focused (e.g., acceptance, positive 
reframing) and problem-focused (e.g., instrumental support, planning) coping, or adaptive coping strategies, are more effective than strategies categorized as dysfunctional or maladaptive (e.g., denial, venting, substance use). Further, the nature of the traumatic event may influence the effectiveness of coping strategies such that temporary losses may benefit from different techniques as compared to more permanent losses or changes (Shing et al. 2016).

In a study of over 5000 adults in Spain during a country-wide COVID-19 lockdown, $65 \%$ of participants reported anxious or depressive symptoms. Lower levels of anxious and/or depressive symptoms were associated with maintaining a healthy diet, avoiding news updates related to COVID-19, talking with friends and relatives, pursuing hobbies, spending time outdoors and following a routine (Fullana et al. 2020). In April 2020 in the U.S., a national sample recruited online via MTurk reported most commonly using distraction, active coping and social support in response to COVID-19. Of note, woman-identified participants were more likely to use multiple emotion-focused strategies, including religious support, whereas participants who were young, financially insecure, or identified as a sexual minority were more likely to engage in maladaptive strategies including disengagement and substance use, as well as humor, an adaptive coping strategy (Park et al. 2020).

\section{Coping Among the (Non)Religious/(Non)Spiritual}

Though the potential benefits of religious and/or spiritual identification and engagement for well-being are well-documented (Paloutzian et al. 2013), the absence of religious and/or spiritual belief is not necessarily associated with low levels of well-being, or poor adjustment following traumatic events. Rather, the coherency hypothesis suggests the ability to make sense of the world, in whatever way fits for a person, is associated with well-being. Among nonreligious and nonspiritual people in Canada, engagement in religiousness, spirituality, and related practices (e.g., meditation, church attendance) ranged from not associated to negatively associated with mental health, such that higher R/S among nonreligious and nonspiritual participants was related to lower well-being (Speed et al. 2021). Therefore, it is more accurate to suggest religious and spiritual engagement (e.g., prayer, church attendance) is generally beneficial for the health of those with religious and spiritual beliefs and values (Speed et al. 2019), but not a necessary condition of health for all people. Galen et al. (2011), for example, found evidence of a curvilinear relationship between (non)religiousness and well-being, such that those with high levels of religiousness/spirituality as well as those with high levels of nonreligiousness/ nonspirituality reported higher well-being as compared to those with medium or low levels of (non)religiousness/(non)spirituality. However, scant literature has explored the implications of this curvilinear relationship for mental health and well-being in response to specific traumatic events (e.g., natural disasters, pandemics).

The authors (Abbott et al. 2021) examined the mediating role of coping strategies in the relationship between trauma intensity and posttraumatic symptoms and growth, as moderated by (non)religiousness/(non)spirituality following a natural disaster in a small, rural city in the Southeastern U.S. Consistent with previous 
research, lower levels of depressive symptoms and higher posttraumatic growth were associated with higher institutional religiousness and individual spirituality. However, upon probing an interaction between trauma and non-religion/non-spirituality, nonreligious and nonspiritual participants reported lower depressive symptoms and more posttraumatic growth when employing adaptive coping strategies, specifically problem-focused coping (e.g., active coping, instrumental support and planning; Abbott et al. 2021). This preliminary study suggests differences, if any, in mental health across the (non)religious/(non)spiritual spectrum may be associated with coping mechanisms utilized and, perhaps, the congruence of coping style with (non)belief system.

\section{The Present Study}

The current study sought to examine the relationships between pandemic-related trauma symptoms, (non)religiousness and (non)spirituality, coping strategies and psychological distress among U.S. Americans. In particular, we were interested in exploring differences, if any, between the coping mechanisms applied by individuals ranging from atheist to very religious and subsequent mental health outcomes, in response to the COVID-19 pandemic. Specifically, we hypothesized that 1) the relationship between pandemic-related trauma and psychological distress would operate indirectly through coping style and 2) the relationships between trauma, psychological distress and coping will be moderated by institutional religiousness and individual spirituality such that differences are observed in those at high and low levels of $\mathrm{R} / \mathrm{S}$, as compared to moderate levels of $\mathrm{R} / \mathrm{S}$, in the use of coping in response to the pandemic and the relationship between coping style and psychological distress (see Fig. 1).

\section{Method}

\section{Participants}

One hundred fifty-three participants (68\% female, $17 \%$ male, $15 \%$ unreported sex assigned at birth) volunteered through social media snowball sampling. The ethnic/ racial composition of the sample was 74\% White, 3\% Black, 7\% Hispanic/Latinx, 10\% Asian/Asian American/Pacific Islander and 1\% Arab/Persian/Middle Eastern. The median educational attainment was Associate's Degree level and the median reported household income was between $\$ 60,000$ and $\$ 99,999$. Participants came from 33 different states with the largest contingents residing in Washington state (20\%), Texas (8\%) and California (7\%). Twenty-nine percent of the sample identified as disabled; specifically, 3\% identified a sensory impairment, $4 \%$ a mobility impairment, $5 \%$ a learning disability and $21 \%$ a mental health disorder. Importantly, $48 \%$ of respondents identified with a Christian faith, while a combined $48 \%$ identified as either atheist (12.5\%), agnostic (12.5\%), or religiously unaffiliated (23\%). Data were collected in the period of time during which the majority of US jurisdictions had 


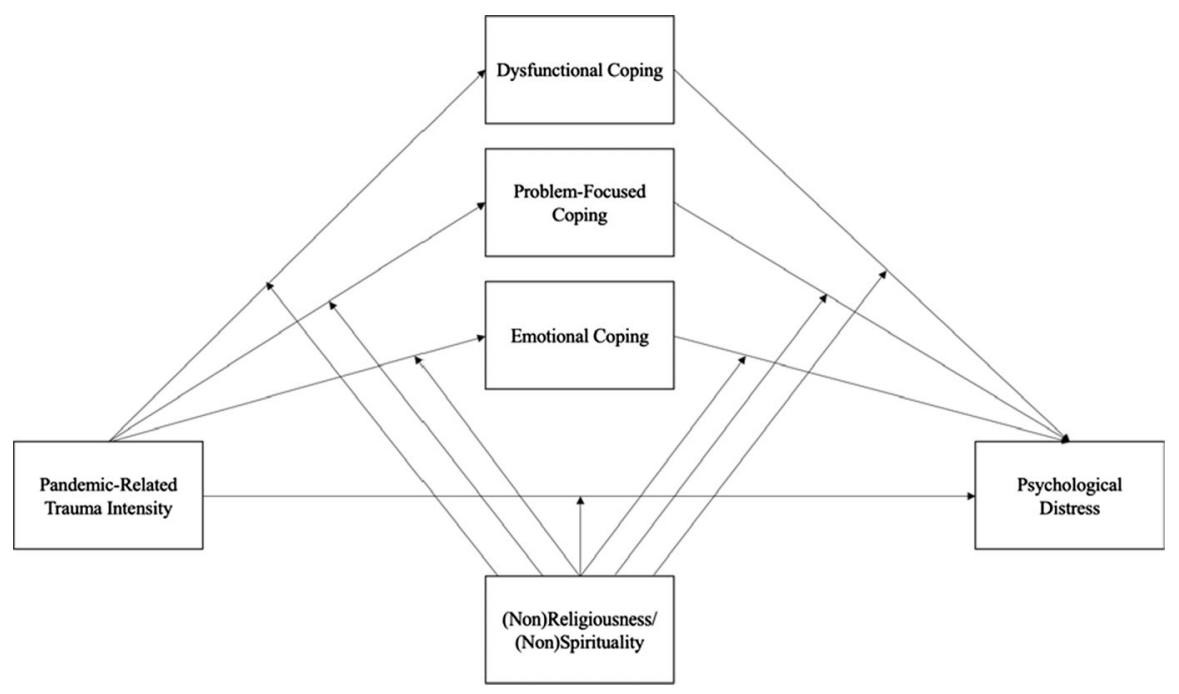

Fig. 1 Hypothesized moderated-mediation model

enacted "Stay At Home" or similar types of orders and recruitment was discontinued when restrictions were relaxed by many state and local governing bodies. A posthoc sensitivity analysis revealed sufficient power for detecting medium-sized effects $\left(f^{2}=0.10\right)$ at standard significance and power criteria $(\alpha=0.05$, power $=0.80)$.

\section{Procedure}

Institutional Review Board approval was provided by Central Michigan University (\#2020-499). Data collection began in late April 2020 and completed in midJune 2020, and the study materials were pre-registered on an open science forum at (https://osf.io/tsdr3/.). Participants were recruited primarily via social media (e.g., Reddit) and snowball sampling. All U.S.-based adults (18 years of age or older) were invited to engage in questions related to their experience of the pandemic (e.g., stress, economic impact, isolation) and (4) surveys: Brief COPE (B-COPE; Carver 1997), Depression, Anxiety, Stress Scales - 21 (DASS-21; Lovibond et al. 1995), NonReligious/NonSpiritual Scale (NRNSS; Cragun et al. 2015) and a demographics questionnaire including items related to social/physical distancing behaviors.

\section{Measures}

\section{Coping Strategies}

Coping strategies utilized by participants were assessed using the 28-item B-COPE (Carver 1997). The scale is comprised of 14 subscales that may be organized into three composite subscales. The acceptance, emotional support, humor, positive 
reframing and religion subscales (Emotion-Focused) and active coping, instrumental support and planning subscales (Problem-Focused) are categorized as Adaptive, whereas the behavioral disengagement, denial, self-distraction, self-blame, substance use and venting subscales (Dysfunctional) are Maladaptive (Carver et al. 1989). Initial reliabilities were good (Emotion-Focused $=0.72$; Problem-Focused $=0.84$; Dysfunctional $=0.75$ ) and construct validity was evidenced (Cooper et al. 2008). In the present study, internal consistency for each broad type of coping were as follows: Problem-Focused Coping, $\alpha=74$; Emotion-Focused Coping, $\alpha=0.58$ and Dysfunctional Coping, $\alpha=74$. The B-COPE is the most frequently used measure of coping across academic journals. Additionally, though the alpha for Emotion-Focused Coping in this study is low, it is within the typical range reported for subscales in other studies using the B-COPE; the two other subscales' alphas are similar to the median in extant literature (Kato 2013).

We additionally asked an open-ended question prompting participants to report any coping behaviors in which they would normally engage but could not during the pandemic. About half of participants (48\%) reported that they were unable to engage in some of their preferred coping behaviors and in all cases, the identified behaviors were emotion-focused. This likely contributed to the low internal consistency for Emotion-Focused Coping. Further, an exploratory reliability analysis with the sample split into high and low (non)religiousness revealed that the emotion-focused coping items had acceptable reliability for participants above the mean in institutional religiousness $(\alpha=0.76)$.

\section{Psychological Distress}

Psychological distress was measured by the Depression, Anxiety, Stress Scales - 21 (DASS-21; Lovibond et al. 1995) consisting of three subscales: Depression, Anxiety and Stress. Participants were asked to report the degree to which statements, including "I found it hard to wind down" and "I felt I had nothing to look forward to," applied to them over the past week on a 4-point Likert scale ranging from "Did not apply to me at all" to "Applied to me very much, most of the time." Higher scores indicate higher rates of depressive, anxious and stress symptoms. Initial reliabilities during development of the scale were $\alpha=0.94$ for the Depression items, $\alpha=0.87$ for the Anxiety items and $\alpha=0.91$ for the Stress items. Reliability for the entire scale in the current study was high $(\alpha=0.94)$.

\section{(Non)Religion/(Non)Spirituality}

(Non)religiosity and (non)spirituality were assessed via the NRNSS (Cragun et al. 2015), a 14-item measure consisting of two subscales: institutional religiousness and individual spirituality. The NRNSS measures religiousness/spirituality on a spectrum ranging from nonreligious/nonspiritual to very religious/spiritual. Participants reported their level of agreement with items including "I would describe myself as a religious person" and "Spirituality is important to me" using a 5-point Likert scale. High scores suggest low institutional religiousness (nonreligiosity) and low individual spirituality 
(nonspirituality) and, inversely, low scores indicate high religiousness and spirituality. Initial testing demonstrated strong internal consistency $(\alpha=0.95)$. In the present study, the $\alpha$ was 0.94 for the nonreligion subscale and 0.93 for the nonspirituality subscale.

\section{Pandemic-Related Trauma}

The intensity of pandemic-related trauma was assessed using 1 item: "Overall, how traumatic is the COVID-19 pandemic for you?". Participants responded using a 7-point Likert scale ranging from "Not at all traumatic" to "Intolerably traumatic." Higher scores indicated participants perceived they were experiencing higher levels of personal trauma due to COVID-19. This measure is consistent with the variable used when testing a similar model in our own previously published research (Abbott et al. 2021).

\section{Results}

The moderated direct and indirect relationships were tested using PROCESS model 59 (Hayes 2013). All effects were computed for each of 10,000 bootstrapped samples and were considered significant if their $95 \%$ confidence intervals did not include zero. For these analyses, pandemic-related trauma intensity was treated as the independent variable, posttraumatic psychological distress was the outcome and each of the three coping styles (problem-focused, emotion-focused, dysfunctional) served as mediators. In the first reported model, the institutional (non)religiousness component of the NRNSS scale was the moderating variable, whereas the second model included the individual (non)spirituality subscale as a moderator (see Fig. 1). For each model, statistical reporting of full model statistics is presented both in main text and tables, while values for unique main and interaction effects are reported in tables only. The analytical plan was designed to examine the most complex relationships possible given expected power limitations. No participants were eliminated from the dataset. However, PROCESS does not impute data. Accordingly, degrees of freedom represent the number of participants who had values for all variables included in the model. The analytical plan was also pre-registered at https://osf.io/tsdr3/. Means, standard deviations and zero-order correlations for all variables are detailed in Table 1 . Hereafter, we will refer to levels of (non)religiousness and (non)spirituality to refer to the spectrum of religious and nonreligious and spiritual and nonspiritual, orientation; low scores indicate high institutional religiousness/individual spirituality, whereas high scores indicate low institutional religiousness/individual spirituality.

\section{Institutional (Non)Religiousness Model}

The combination of trauma, institutional (non)religiousness and the Trauma $\mathrm{x}$ (Non) religiousness interaction predicted significant variance in two of the three types of coping: problem-focused coping, $R^{2}=0.09, F(3,120)=3.93, p=0.01$ and dysfunctional coping, $R^{2}=0.09, F(3,120)=3.93, p=0.01$. Significant unique predictors included (non)religiousness, which was negatively associated with reduced 
Table 1 Descriptive statistics, correlation coefficients and significance values

\begin{tabular}{|c|c|c|c|c|c|c|c|c|}
\hline Variable & $M(S D)$ & 1 & 2 & 3 & 4 & 5 & 6 & 7 \\
\hline 1. Trauma & $2.71(1.26)$ & - & & & & & & \\
\hline $\begin{array}{l}\text { 2. (Non) } \\
\text { Religion }\end{array}$ & $29.23(9.15)$ & .009 & - & & & & & \\
\hline $\begin{array}{l}\text { 3. (Non)Spir- } \\
\text { ituality }\end{array}$ & $23.30(8.21)$ & -.067 & $.598^{* *}$ & - & & & & \\
\hline $\begin{array}{l}\text { 4. Problem- } \\
\text { FocusedCop- } \\
\text { ing }\end{array}$ & $2.32(0.62)$ & $.253^{* *}$ & -.146 & $-.211^{*}$ & - & & & \\
\hline $\begin{array}{l}\text { 5. Emotion- } \\
\text { Focused } \\
\text { Coping }\end{array}$ & $2.44(0.44)$ & .009 & $-.249^{* *}$ & -.132 & $.465^{* *}$ & - & & \\
\hline $\begin{array}{l}\text { 6. Dysfunc- } \\
\text { tional } \\
\text { Coping }\end{array}$ & $1.95(0.36)$ & $.315^{* *}$ & .103 & .077 & .011 & .033 & - & \\
\hline $\begin{array}{l}\text { 7. Depression, } \\
\text { Anxiety, \& } \\
\text { Stress }\end{array}$ & $41.95(12.24)$ & $.302^{* *}$ & .109 & -.021 & -.156 & -.090 & $.632^{* *}$ & - \\
\hline
\end{tabular}

$* * p<.01 ; * p<.05$

problem-focused and emotion-focused coping and pandemic-related trauma, which was positively associated with more dysfunctional coping. The overall model was not significant for emotion-focused coping, $R^{2}=0.05, F(3,120)=2.29, p=0.08$. See Table 2 for summaries of unique effects of predictors on mediators.

The combination of trauma, (non)religiousness, problem-focused coping, emotion-focused coping, dysfunctional coping, Trauma x (Non)religiousness, Problem-focused Coping x (Non)religiousness, Emotion-focused Coping x (Non)

Table 2 Effects of trauma and (non)religion on coping styles

\begin{tabular}{lrrccc}
\hline Variable & \multicolumn{1}{c}{$t$} & \multicolumn{1}{c}{$b$} & $95 \%$ CI Low & $95 \%$ CI Upper & $p$ \\
\hline M1: Problem- Focused Coping & $R^{2}=.09$ & $F=3.93$ & $p=.01$ & & \\
Trauma & 1.92 & 0.62 & -0.79 & 0.79 & .057 \\
(Non)Religion & -2.45 & -0.11 & -0.02 & 1.26 & .016 \\
Trauma x (Non)Religion & 1.13 & 0.04 & -0.03 & -0.03 & .258 \\
M2: Emotion- Focused Coping & $R^{2}=.05$ & $F=2.29$ & $p=.082$ & & \\
Trauma & 1.39 & 0.41 & -0.17 & 1.00 & .166 \\
(Non)Religion & -2.05 & -0.08 & -0.16 & -0.01 & .043 \\
Trauma x (Non)Religion & -1.10 & -0.04 & -0.10 & 0.03 & .272 \\
M3: Dysfunctional Coping & $R^{2}=.09$ & $F=3.93$ & $p=.01$ & & \\
Trauma & $2 . .66$ & 0.71 & 0.18 & 1.24 & .009 \\
(Non)Religion & 1.46 & 0.05 & -0.02 & 0.12 & .148 \\
Trauma x (Non)Religion & 1.21 & 0.04 & -0.02 & 0.10 & .227 \\
\hline
\end{tabular}


religiousness and Dysfunctional Coping $\mathrm{x}$ (Non)religiousness predicted significant variance in psychological distress, $R^{2}=0.54, F(9,114)=14.65, p<0.001$. Significant unique predictors included trauma, problem-focused coping and dysfunctional coping. Increased trauma and dysfunctional coping were each associated with increased psychological distress, while increased problem-focused coping predicted lower distress. See Table 3 for a summary of the effects of unique predictors on the outcome variable.

While the interaction between trauma and (non)religiousness was nonsignificant, indicating it cannot be concluded that individuals were affected by trauma differently based on their level of institutional (non)religiousness, the conditional direct effect of trauma on psychological distress was significant at low and moderate levels of institutional (non)religiousness but not significant at high levels of (non)religiousness. However, the conditional indirect effect of trauma on psychological distress through the mechanism of dysfunctional coping was significant at high nonreligiousness and moderate levels of (non)religiousness, but not at low levels of (non) religiousness.

Trauma and dysfunctional coping were both significant predictors of psychological distress and the trauma effect was significant via the mechanism of dysfunctional coping at moderate and high levels of (non)religiousness. This indicates, perhaps, that both religious and nonreligious individuals experienced psychological distress in the aftermath of trauma but that nonreligious individuals experienced psychological distress due to utilization of dysfunctional coping strategies. The question of whether the direct and indirect effects are different for those across the spectrum of institutional (non)religiousness remains unclear with current statistical power, however. See Table 3 for summaries of unique effects of predictors on depressive symptoms.

Table 3 Effects of trauma, (non)religion, coping and interactions on psychological distress

\begin{tabular}{|c|c|c|c|c|c|}
\hline $\begin{array}{l}\text { Y: Psychological Distress } \\
\text { Variable }\end{array}$ & $\begin{array}{l}R^{2}=.54 \\
t\end{array}$ & $\begin{array}{l}F=14.66 \\
B\end{array}$ & $\begin{array}{l}p<.001 \\
95 \% \text { CI Low }\end{array}$ & 95\% CI Upper & $p$ \\
\hline Trauma & 3.04 & 0.10 & 0.03 & 0.16 & .003 \\
\hline Problem-Focused Coping & -2.68 & -0.03 & -0.05 & -0.01 & .008 \\
\hline Emotion-Focused Coping & 0.96 & 0.01 & -0.01 & 0.03 & .337 \\
\hline Dysfunctional Coping & 8.08 & 0.09 & 0.07 & 0.11 & .001 \\
\hline (Non)Religion & 0.32 & 0.00 & -0.01 & 0.01 & .754 \\
\hline Trauma $\mathrm{x}$ (Non)Religion & -0.75 & -0.003 & -0.01 & 0.004 & .454 \\
\hline $\begin{array}{l}\text { Problem-Focused Coping x } \\
\text { (Non)Religion }\end{array}$ & -0.59 & -0.001 & -0.003 & 0.001 & .552 \\
\hline $\begin{array}{l}\text { Emosion-focused Coping x } \\
\text { (Non)Religion }\end{array}$ & -1.19 & -0.001 & -0.004 & 0.001 & .236 \\
\hline $\begin{array}{l}\text { Dysffunctional Coping x } \\
\text { (Non)Religion }\end{array}$ & -1.22 & -0.002 & -0.003 & 0.001 & .225 \\
\hline
\end{tabular}




\section{Individual (Non)Spirituality Model}

The combination of trauma, (non)spirituality and the Trauma $\mathrm{x}$ (Non)spirituality interaction predicted significant variance in two of the three types of coping: Problem-focused coping, $R^{2}=0.08, F(3,119)=3.47, p=0.02$ and dysfunctional coping, $R^{2}=0.08, F(3,119)=3.22, p=0.03$. The overall model was not significant for emotion-focused coping, $R^{2}=0.02, F(3,119)=0.80, p=0.50$. Significant unique predictors included higher levels of (non)spirituality associated with reduced problem-focused coping and higher levels of pandemic-related trauma associated with higher levels of dysfunctional coping. See Table 4 for summaries of unique effects of predictors on mediators.

The combination of trauma, (non)spirituality, problem-focused coping, emotionfocused coping, dysfunctional coping, Trauma x (Non)spirituality, Problem-focused Coping x (Non)spirituality, Emotion-focused Coping x (Non)spirituality and Dysfunctional Coping $\mathrm{x}$ (Non)spirituality predicted significant variance in psychological distress, $R^{2}=0.54, F(9,113)=14.59, p<0.001$. Significant unique predictors included trauma, problem-focused coping, dysfunctional coping and the (Non)spirituality x Dysfunctional Coping interaction. Increased trauma and dysfunctional coping were both associated with higher psychological distress, whereas increased problem-focused coping predicting lower depressive symptoms. See Table 5 for summaries of unique effects of predictors on the outcome variable.

Probing the (Non)spirituality x Dysfunctional Coping interaction showed that the indirect effect of trauma on psychological distress through the mechanism of dysfunctional coping was significant at moderate and high levels of (non)spirituality but not at low levels of (non)spirituality. This is consistent with the nonsignificant trend found in the (non)religiousness model reported above and lends credence to the assumption that the interaction in that model should reach significance with more statistical power.

Table 4 Effects of trauma and (non)spirituality on coping styles

\begin{tabular}{|c|c|c|c|c|c|}
\hline Variable & $T$ & $B$ & $95 \%$ CI Low & 95\% CI Upper & $p$ \\
\hline M1: Problem Focused Coping & $R^{2}=.08$ & $F=3.48$ & $p=.02$ & & \\
\hline Trauma & 1.77 & 0.58 & -0.07 & 1.23 & .079 \\
\hline (Non)Spirituality & -2.20 & -0.11 & -0.21 & -0.01 & .030 \\
\hline Trauma x (Non)SpIrituality & 0.97 & 0.04 & -0.04 & 0.11 & .332 \\
\hline M2: Emotion Focused Coping & $R^{2}=.02$ & $F=0.80$ & $p=.497$ & & \\
\hline Trauma & 1.15 & 0.35 & -0.25 & 0.95 & .252 \\
\hline (Non)Spirituality & -0.91 & -0.04 & -0.13 & 0.05 & .366 \\
\hline Trauma x (Non)Sprituality & -0.24 & -0.01 & -0.08 & 0.06 & .810 \\
\hline M3: Dysfunctional Coping & $R^{2}=.08$ & $F=3.22$ & $p=.03$ & & \\
\hline Trauma & $2 . .94$ & 0.79 & 0.26 & 1.32 & .004 \\
\hline (Non)Spirituality & 0.93 & 0.04 & -0.04 & 0.12 & .356 \\
\hline Trauma x (Non)Spirituality & 0.51 & 0.02 & -0.04 & 0.08 & .609 \\
\hline
\end{tabular}


Table 5 Effects of trauma, (non)spirituality, coping and interactions on psychological distress

\begin{tabular}{|c|c|c|c|c|c|}
\hline $\begin{array}{l}\text { Y: Psychological Distress } \\
\text { Variable }\end{array}$ & $\begin{array}{l}R^{2}=.54 \\
T\end{array}$ & $\begin{array}{l}F=14.59 \\
b\end{array}$ & $\begin{array}{l}p<.001 \\
95 \% \text { CI Low }\end{array}$ & 95\% CI Upper & $p$ \\
\hline Trauma & 2.93 & 0.10 & 0.03 & 0.16 & .004 \\
\hline Problem-Focused Coping & -3.11 & -0.03 & -0.05 & -0.01 & .002 \\
\hline Emotion-Focused Coping & 1.53 & 0.02 & -0.004 & 0.04 & .127 \\
\hline Dysfunctional Coping & 8.11 & 0.09 & 0.07 & 0.11 & .001 \\
\hline (Non)Spirituality & -1.16 & -0.006 & -0.02 & 0.004 & .248 \\
\hline Trauma x (Non)Spirituality & 0.72 & -0.003 & -0.005 & 0.01 & .471 \\
\hline $\begin{array}{l}\text { Problem-Focused Coping x } \\
\text { (Non)Spirituality }\end{array}$ & -0.77 & -0.001 & -0.003 & 0.001 & .445 \\
\hline $\begin{array}{l}\text { Emotion-Focused Coping } \mathrm{x} \\
\text { (Non)Spirituality }\end{array}$ & -0.02 & 0.000 & -0.003 & 0.003 & .987 \\
\hline $\begin{array}{l}\text { Dysfunctional Coping } \mathrm{x} \\
\text { (Non)Spirituality }\end{array}$ & -2.21 & -0.003 & -0.006 & -0.001 & .029 \\
\hline
\end{tabular}

\section{Discussion}

Overall, analyses suggested our general hypothesis that the relationship between pandemic-related trauma and psychological distress would operate indirectly through coping style and the relationships between trauma, psychological distress and coping would be moderated by institutional religiousness and individual spirituality held, but in a way that seemed appropriately unique for the COVID-19 pandemic. The pandemic and related social distancing policies likely reduced participants' ability to engage in emotion-focused coping (e.g., emotional support, religion) and decreased the internal reliability of the emotion-focused coping subscale of the B-COPE. Perhaps due to the inability to engage in emotion-focused coping, individuals became more reliant on dysfunctional coping strategies, which led to more psychological distress (particularly for those higher in nonreligiousness/nonspirituality). Nonreligious individuals reported significantly lower likelihood of engaging in more adaptive coping strategies, and although there was not a significant difference in reported use of dysfunctional coping across (non)religiousness/ (non)spirituality, higher levels of dysfunctional coping were associated with higher levels of psychological distress for those at higher levels of (non)religiousness and (non)spirituality.

\section{Integration with Previous Research}

The relationship between pandemic-related trauma and psychological distress in the current examination is congruent with previous collective trauma research (Baral et al. 2019; Goldmann et al. 2014), including a similar examination of coping and (non)religiousness following a natural disaster (Abbott et al. 2021) and emerging data related to COVID-19 (Schecter et al. 2020). Therefore, it appears depressive, 
anxious and stress symptoms are possible in response to the COVID-19 pandemic among people in the US across the (non)religious/(non)spiritual spectrum.

Evidence regarding the relationships between religiousness and health is mixed. Some research has suggested a curvilinear relationship between (non)religiousness and health (Galen 2018), but assumptions of compromised health among nonreligious people persist, in part due to an abundance of scholarship demonstrating a positive relationship between religiousness and psychological health (Paloutzian et al. 2013). More recent research found comparable health outcomes and levels of happiness among theists and nontheists (Speed et al. 2019) and emphasized the importance of a coherent sense of the world and congruence between (non)religiousness/(non)spirituality and behaviors for health outcomes (Speed et al. 2021). Our results may speak to some nuances in this relationship and the contexts in which it operates. First, our results are consistent with prior findings indicating that those with moderate levels of religiousness exhibit lower well-being than their highly religious or nonreligious counterparts (Galen et al. 2011) and that this may be related to dysfunctional coping. Second, the unique context of the COVID-19 pandemic, associated stay-at-home orders and the resulting physical distancing recommendations may have exacerbated social isolation among nonbelievers and prevented them from engaging in effective emotion-focused coping strategies-as evidenced by the low reliability of the emotion-focused coping items for nonreligious individuals and the open-ended responses.

Internal consistency for the emotion-focused coping subscale was adequate among more religious participants. Indeed, religious/spiritual participants could still engage in independent faith-based coping strategies including prayer, meditation and comfort in their belief system and places of religious worship were among the first locations allowed to reopen in many areas of the country. Although nonreligious people benefit similarly from social connection with like-minded people (Galen 2015), organized groups of nonbelievers can be difficult to find, inaccessible to nonbelievers with marginalized cultural positionalities (Abbott et al. 2020), or primarily online where the benefits of engagement on mental health may be less pronounced (Brewster et al. 2020) even under normal circumstances. All of this is consistent with the notion that social support and belonging are the driving mechanisms behind the well-documented relationship between religious commitment and well-being.

Further, although previous research suggested nonreligious/nonspiritual people were more likely to use problem-focused coping in response to collective trauma in the form of a natural disaster (Abbott et al. 2021), perhaps due to their tendency to engage in analytical thinking to evaluate circumstances (Franks et al. 2017; Baker et al. 2012), most people are likely able to draw upon knowledge of other natural disasters by which they or others have been impacted in determining a personal response. The novel nature of the COVID-19 pandemic may have made it difficult for nonreligious people to engage in otherwise preferred and problem-focused methods of coping like planning, taking action to improve the situation and soliciting advice from others. Substance use, a dysfunctional coping strategy, is associated with COVID-related stress, particularly among women (Rodriguez et al. 2020) and represents a possible explanation for the relationship between higher trauma 
intensity and higher use of dysfunctional coping in the current investigation given a little over two-thirds of participants were women.

\section{Limitations and Future Directions}

The current study was limited by having insufficient statistical power for detecting small effects. The limited time frame for collecting data, during the time period during which US jurisdictions enacted the most restrictive measures to contain the novel coronavirus and a lack of funding for quickly recruiting large numbers of participants resulted in a study powered enough to detect medium-sized effects and larger. As only 60 participants out of the already modest sample agreed to be contacted for follow-up, we were unable to conduct a pre-registered follow-up data collection phase meant to measure posttraumatic growth. Future research examining differences in coping strategies in response to traumatic events among individuals across the (non)religious/(non)spiritual spectrum should seek larger samples, employ longitudinal methods and explore other relevant variables (e.g., posttraumatic growth) given this and previous research (e.g., Abbott et al. 2021) have demonstrated important differences between individuals high and low on such measures.

Like all COVID-19 research conducted with a nationwide sample, this study was also subject to uncontrolled variance due to the US' lack of a coordinated national response to the pandemic. Restrictions varied greatly state-by-state and sometimes county-by-county. Some states also lagged far behind others in terms of the timing and severity of the pandemic's effects on public health and the economy. Future research should, if possible, control for these factors perhaps using geographically nested analyses or by concentrating on a specific geographic region.

An error in survey design led to the omission of a measure for participant age. Age effects would be hard to find in this study due to the relatively small sample size and the use of a sampling method (e.g., Reddit) that would likely result in a very young sample. A majority of young adults, however, are living with their parents rather than cohabitating with a friend, spouse, or significant other during the COVID-19 pandemic (Fry et al. 2020), which could, along with the increased propensity for the young to be non-religious (Pew Research Center 2019), make it more difficult for them to engage in emotion-focused coping during a socially isolating event like a pandemic. Future research should also examine age-related differences in coping styles and outcomes during the coronavirus era.

\section{Conclusion}

The present examination suggests a complex relationship between self-perceived COVID-19-related trauma intensity, coping strategies, psychological distress and (non)religiousness/(non)spirituality, such that people across the (non)religious/(non) spiritual spectrum experience distress related to the COVID-19 pandemic. However, the use and availability of typical adaptive and maladaptive coping mechanisms 
appears to vary by strength of (non)religious/(non)spiritual orientation in response to collective trauma. Future, large-scale studies are needed to continue exploring the relationship between exposure to collective traumatic events, psychological health and coping among people of diverse (non)religious and (non)spiritual identities in the interest of understanding what adaptive coping strategies are most useful, particularly for nontheists given their underrepresentation in extant trauma and coping literature.

Authors' Contributions Drs. Franks and Abbott collaborated on the development of the study including research design, data collection and manuscript development. Dr. Abbott was primarily responsible for drafting the manuscript, whereas Dr. Franks was primarily responsible for executing statistical analyses and drafting the results section. Both authors reviewed and approved the final manuscript.

Data Availability The study materials were pre-registered on an open science forum and data is available upon request from the authors.

\section{Declarations}

Conflicts of interest The authors have no conflicts of or competing interests to disclose.

Informed consent The study was approved by the Institutional Review Board (IRB) at Central Michigan University (\#2020-499). Informed consent was obtained from all participants prior to participation in the study.

\section{References}

Abbott, D. M., Franks, A. S., Cook, C., \& Mercier, C. (2021). (Non)religious coping with a natural disaster in a rural U.S. community. Secularism and Nonreligion. 10(1), 1-14. https://doi.org/https://doi. org/10.5334/snr.139

Abbott, D. M., Mollen, D., Mercier, C., Anaya, E. J., \& Rukus, V. A. (2020). "Isn't atheism a White thing?": Centering the voices of atheists of color. Journal of Counseling Psychology, 67(3), 275287. https://doi.org/10.1037/cou0000399

Aten, J. D., Smith, W. R., Davis, E. B., Van Tongeren, D. R., Hook, J. N., Davis, D. E., Shannonhouse, L., DeBlaere, C., Ranter, J., O’Grady, K., \& Hill, P. C. (2019). The psychological study of religion and spirituality in a disaster context: A systematic review. Psychological Trauma: Theory, Research, Practice and Policy, 11(6), 597-613. https://doi.org/10.1037/tra0000431

Baker, M. J., \& Robbins, M. (2012). American on-line atheists and psychological type. Mental Health, Religion, \& Culture, 15(10), 1077-1084. https://doi.org/10.1080/13674676.2012.707433.

Baral, I. A., \& Bhagawati, K. C. (2019). Posttraumatic stress disorder and coping strategies among adult survivors of earthquake. Nepal. BMC Psychiatry, 19(1), 118-127. https://doi.org/10.1186/ s12888-019-2090-y

Blashill, A. J., Perry, N., \& Safren, S. A. (2011). Mental health: A focus on stress, coping and mental illness as it relates to treatment retention, adherence and other health outcomes. Current HIV/AIDS Reports, 8(4), 215-222. https://doi.org/10.1007/s11904-011-0089-1

Bonanno, G. A. (2004). Loss, trauma and human resilience: Have we underestimated the human capacity to thrive after extremely aversive events? American Psychologist, 59(1), 20-28. https://doi.org/10. 1037/0003-066X.59.1.20

Bonanno, G. A., Brewin, C. R., Kaniasty, K., \& Greca, A. M. L. (2010). Weighing the costs of disaster: Consequences, risks and resilience in individuals, families and communities. Psychological Science in the Public Interest, 11(1), 1-49. https://doi.org/10.1177/1529100610387086 
Brewster, M. E., Velez, B. L., Geiger, E. F., \& Sawyer, J. A. (2020). It's like herding cats: Atheist minority stress, group involvement and psychological outcomes. Journal of Counseling Psychology., 67(1), 1-13. https://doi.org/10.1037/cou0000392

Carver, C. S. (1997). You want to measure coping but your protocol's too long: Consider the brief COPE. International Journal of Behavioral Medicine, 4(1), 92-100. https://doi.org/10.1207/s15327558i jbm0401_6

Carver, C. S., Scheier, M. F., \& Weintraub, J. K. (1989). Assessing coping strategies: A theoretically based approach. Journal of Personality and Social Psychology, 56(2), 267-283. https://doi.org/10. 1037/0022-3514.56.2.267

Cherry, K. E., Lyon, B. A., Sampson, L., Galea, S., Nezat, P. F., \& Marks, L. D. (2017). Prior hurricane and other lifetime trauma predict coping style in older commercial fishers after the BP Deepwater Horizon oil spill. Journal of Applied Biobehavioral Research, 22(2), e12058. https://doi.org/10. 1111/jabr. 12058

Comas-Díaz, L., Hall, G. N., \& Neville, H. A. (2019). Racial trauma: Theory, research and healing: Introduction to the special issue. American Psychologist, 74(1), 1-5. https://doi.org/10.1037/amp00 00442

Cooper, C., Katona, C., \& Livingston, G. (2008). Validity and reliability of the Brief COPE in carers of people with dementia. The Journal of Nervous and Mental Disease, 196(11), 838-843. https://doi. org/10.1097/nmd.0b013e31818b504c

Cragun, R. T., Hammer, J. H., \& Nielsen, M. (2015). The nonreligious-nonspiritual scale (NRNSS): Measuring everyone from atheists to zionists. Science, Religion and Culture, 2(3), 36-53. https:// doi.org/https://doi.org/10.17582/journal.src/2015/2.3.36.53

Duane, A. M., Stokes, K. L., DeAngelis, C. L., \& Bocknek, E. L. (2020). Collective trauma and community support: Lessons from Detroit. Psychological Trauma: Theory, Research, Practice and Policy, 12(5), 452-454. https://doi.org/10.1037/tra0000791

Erickson, K. T. (1976). Everything in its path: Destruction of community in the Buffalo Creek flood. Simon \& Schuster.

Franks, A. S., \& Scherr, K. C. (2017). Analytic thinking reduces anti-atheist bias in voting intentions. The International Journal for the Psychology of Religion, 27(3), 129-140. https://doi.org/10.1080/10508 619.2017.1313013

Fry, R., Passell, J. S., \& Cohn, D. (2020). A majority of young adults in the U.S. live with their parents for the first time since the Great Depression. https://www.pewresearch.org/fact-tank/2020/09/04/amajority-of-young-adults-in-the-u-s-live-with-their-parents-for-the-first-time-since-the-great-depre ssion/

Fullana, M. A., Hidalgo-Mazzei, D., Vieta, E., \& Radua, J. (2020). Coping behaviors associated with decreased anxiety and depressive symptoms during the COVID-19 pandemic and lockdown. Journal of Affective Disorders, 275, 80-81. https://doi.org/10.1016/j.jad.2020.06.027

Galen, L. W. (2015). Atheism, wellbeing and the wager: Why not believing in God (with others) is good for you. Science, Religion and Culture, 2(3), 54-69. https://doi.org/https://doi.org/10.17582/journal. $\mathrm{src} / 2015 / 2.3 .54 .69$

Galen, L. W. (2018). Focusing on the nonreligious reveals secular mechanisms underlying well-being and prosociality. Psychology of Religion and Spirituality, 10(3), 296-306. https://doi.org/10.1037/rel00 00202

Galen, L. W., \& Kloet, J. D. (2011). Mental well-being in the religious and the non-religious: Evidence for a curvilinear relationship. Mental Health, Religion and Culture, 14(7), 673-689. https://doi.org/ 10.1080/13674676.2010.510829

Goldmann, E., \& Galea, S. (2014). Mental health consequences of disasters. Annual Review of Public Health, 35(1), 169-183. https://doi.org/10.1146/annurev-publhealth-032013-182435

Hayes, A. F. (2013). Introduction to mediation, moderation and conditional process analysis: A regression-based approach. The Guilford Press.

Hwang, K., Hammer, J. H., \& Cragun, R. T. (2011). Extending religion-health research to secular minorities: Issues and concerns. Journal of Religion and Health, 50(3), 608-622. https://doi.org/10.1007/ s10943-009-9296-0

Kato, T. (2013). Frequently used coping scales: A meta-analysis. Stress \& Health, 31(4), 315-323. https://doi.org/10.1002/smi.2557

Kelly, M., Lubitow, A., Town, M., \& Mercier, A. (2020). Collective trauma in queer communities. Sexuality \& Culture. Advance Online Publication. https://doi.org/10.1007/s12119-020-09710-y

Lazarus, R. S., \& Folkman, S. (1984). Stress, appraisal, and coping. Springer. 
Lei, M.-K., \& Klopack, E. T. (2020). Social and psychological consequences of the COVID-19 outbreak: The experiences of Taiwan and Hong Kong. Psychological Trauma: Theory, Research, Practice, and Policy, 12(S1), S35-S37. https://doi.org/10.1037/tra0000633

Lovibond, S. H., \& Lovibond, P. F. (1995). Manual for the Depression Anxiety Stress Scales (2 ${ }^{\text {nd }}$ ed.). Psychology Foundation.

Luszczynska, A., Benight, C. C., \& Cieslak, R. (2009). Self-efficacy and health-related outcomes of collective trauma: A systematic review. European Psychologist, 14(1), 51-62. https://doi.org/10.1027/ 1016-9040.14.1.51

Neria, Y., Nandi, A., \& Galea, S. (2008). Post-traumatic stress disorder following disasters: A systematic review. Psychological Medicine, 38(4), 467-480. https://doi.org/10.1017/S0033291707001353

Paloutzian, R. F., \& Park, C. L. (2013). Handbook of the psychology of religion and spirituality. The Guilford Press.

Park, C. L., Russell, B. S., Fendrich, M., Finkelstein-Fox, L., Hutchinson, M., \& Becker, J. (2020). Americans' COVID-19 stress, coping and adherence to CDC guidelines. Journal of General Internal Medicine. Advance online publication. https://doi.org/10.1007/s11606-020-05898-9

Pew Research Center. (2019). In U.S., decline of Christianity continues at rapid pace: An update on America's changing religious landscape. https://www.pewforum.org/2019/10/17/in-u-s-decline-ofchristianity-continues-at-rapid-pace/

Rodriguez, L. M., Litt, D. M., \& Stewart, S. H. (2020). Drinking to cope with the pandemic: The unique associations of COVID-19-related perceived threat and psychological distress to drinking behaviors in American men and women. Addictive Behaviors, 110, 106532. https://doi.org/10.1016/j.addbeh. 2020.106532

Schechter, A., Diaz, F., Moise, N., Antsey, D. E., Ye, S., Agarwal, S., Birk, J. L., Brodie, D., Cannone, D. E., Chang, B., Claassen, J., Cornelius, T., Derby, L., Dong, M., Givens, R. C., Hochman, B., Homma, S., Kronish, I. M., Lee, S. A., \& J.,...Abdalla, M. . (2020). Psychological distress, coping behaviors, and preferences for support among New York healthcare workers during the COVID-19 pandemic. General Hospital Psychiatry, 66, 1-8. https://doi.org/10.1016/j.genhosppsych.2020.06. 007

Shing, E. Z., Jayawickreme, E., \& Waugh, C. E. (2016). Contextual positive coping as a factorcontributing to resilience after disasters. Journal of Clinical Psychology, 72(12), 1287-1306. https://doi.org/ $10.1002 /$ jclp. 22327

Silver, R. C., \& Updegraff, J. A. (2013). Searching for and finding meaning following personal and collective traumas. In K. D. Markman, T. Proulx, \& M. J. Lindberg (Eds.), The psychology of meaning (pp. 237-255). https://doi.org/10.1037/14040-012

Speed, D., \& Hwang, K. (2019). Heretic, heal thyself! Atheism, nonreligion and health. Psychology of Religion and Spirituality, 11(3), 297-307. https://doi.org/10.1037/re10000158

Speed, D., \& Fowler, K. (2021). One size doesn't fit all: Religious/spiritual identities moderate salutary effects of religion. Mental Health, Religion, \& Culture., 24(2), 117-124.

Publisher's Note Springer Nature remains neutral with regard to jurisdictional claims in published maps and institutional affiliations. 